\title{
ON DISCONJUGATE DIFFERENTIAL EQUATIONS
}

\author{
BY \\ PHILIP HARTMAN $\left(^{1}\right)$
}

Introduction. In Wintner's [16] terminology (when $n=2$ ), a linear differential equation

$$
u^{(n)}+p_{n-1}^{(t)} u^{(n-1)}+\cdots+p_{0}(t) u=0
$$

with continuous coefficients on a $t$-interval $I$ is said to be disconjugate on $I$ if no solution $(\not \equiv 0)$ has $n$ zeros on $I$. For $n>2$, most known results giving conditions which assure that $(0.1)$ is disconjugate concern perturbations of $u^{(n)}=0$ either for a fixed finite interval [as, e.g., in the theorem of de la Vallee Poussin (cf. [2, Exercise 5.3(d), p. 346])] or for large $t$. This paper deals with equations (1.1) which are perturbations of equations with constant coefficients, disconjugate on $-\infty<t<\infty$. As corollaries, we obtain theorems which are refinements of known results concerning perturbations of $u^{(n)}=0$, but we do not obtain the "best" constants occurring in some of these results $(n=2)$.

The proofs depend on the technique introduced in [5] for discussing asymptotic behavior of solutions of perturbed linear systems with constant coefficients (cf. [2, Chapter X]). This technique is based on suitable changes of variables and arguments which have been subsumed by general theorems of Ważewski [15], (cf. [2, pp. 278-283]). $\S \S 1$ and 2 use the simple Lemma 4.2, [2, p. 285]; $\S 3$ requires a generalization given as Theorem $\left({ }^{*}\right)$ in an Appendix below.

In addition to arguments from the theory of asymptotic integration, the proofs use a theorem of Pólya [14] characterizing equations (0.1) disconjugate on an open interval $I$ in terms of Wronskians of subsets of solutions of $(0.1)$; for a generalization, see [2, pp. 51-54] (also obtained in [6]). Theorems $I_{* *}$ and IV of Pólya [14] show that no solution $(\not \equiv 0)$ of $(0.1)$ on an open interval $I$ has $n$ distinct zeros if and only if no solution $(\not \equiv 0)$ has $n$ zeros counting multiplicities; cf. also [13]. (A generalization of this last fact, when the linear family of solutions of $(0.1)$ is replaced by an arbitrary (not necessarily linear) interpolating family of functions, is given in [1].)

In $\S 4$, it is observed that the results of the previous sections, together with theorems and methods of Lasota and Opial [8], give criteria for the existence of solutions of certain nonlinear boundary value problems.

Received by the editors June 27, 1967.

(1) Research partially sponsored by the Air Force Office of Scientific Research, Office of Aerospace Research, United States Air Force, under AFOSR Contract No. AF 49(638)-1382. 
1. Distinct real roots. We shall first consider linear differential equations

$$
u^{(n)}+a_{n-1} u^{(n-1)}+\cdots+a_{0} u=q_{n-1}(t) u^{(n-1)}+\cdots+q_{0}(t) u
$$

which are perturbations of equations

$$
u^{(n)}+a_{n-1} u^{(n-1)}+\cdots+a_{0} u=0
$$

with constant coefficients, having real distinct characteristic values.

Assumption $\left(\mathrm{A}_{1}\right)$. Let $a_{0}, \ldots, a_{n-1}$ be $n$ real numbers such that the polynomial

$$
\lambda^{n}+a_{n-1} \lambda^{n-1}+\cdots+a_{0}=0
$$

has real distinct roots $\lambda_{1}<\lambda_{2}<\cdots<\lambda_{n}$ and let

$$
c=\min \left(\lambda_{2}-\lambda_{1}, \ldots, \lambda_{n}-\lambda_{n-1}\right)>0 \text {. }
$$

THEOREM 1.1. Let Assumption $\left(\mathrm{A}_{1}\right)$ hold. Then there exists a positive number $\eta_{0}$, depending on $\lambda_{1}, \ldots, \lambda_{n}$ but independent of $T$, with the following properties: If $q_{0}(t), \ldots, q_{n-1}(t)$ are continuous functions on $0 \leqq t<T(\leqq \infty)$ [or on $0 \leqq t \leqq T(<\infty)$ ] such that

$$
q(t)=\left(\sum_{j=0}^{n-1}\left|q_{j}(t)\right|^{2}\right)^{1 / 2} \geqq 0
$$

satisfies

$$
\int_{0}^{T} q(s) d s<\eta_{0} \text { or } q(t)<c \eta_{0}
$$

or, more generally,

$$
\int_{0}^{t} q(s) e^{-c(t-s)} d s, \quad \int_{t}^{T} q(s) e^{-c(s-t)} d s \leqq \eta<\eta_{0} .
$$

Then (1.1) is disconjugate on $0 \leqq t<T(\leqq \infty)$ [or on $0 \leqq t \leqq T(<\infty)$ ].

Proof. Let $y=\left(u, u^{\prime}, \ldots, u^{(n-1)}\right)$ and write (1.1) as a linear first order system

$$
y^{\prime}=(A+Q(t)) y,
$$

where $A=\left(a_{j k}\right)$ is the constant $n \times n$ matrix with the first $n-1$ rows given by $a_{j k}=1$ or $a_{j k}=0$ for $1 \leqq j \leqq n-1, k=j+1$ or $k \neq j+1$ and the last row is $\left(-a_{0}, \ldots\right.$, $\left.-a_{n-1}\right)$, and $Q(t)$ is the matrix with 0 in the first $n-1$ rows and the last row is $\left(q_{0}(t), \ldots, q_{n-1}(t)\right)$.

Let $\Lambda=\Lambda_{n}=\left(\lambda_{j k}\right)$ be the constant $n \times n$ matrix with $\lambda_{j k}=\lambda_{k}^{j-1}$ for $j, k=1, \ldots, n$. Since the $k$ th column $\left(1, \lambda_{k}, \ldots, \lambda_{k}^{n-1}\right)$ of $\Lambda$ is an eigenvector of $A$ belonging to the eigenvalue $\lambda_{k}$, we have

$$
\Lambda^{-1} A \Lambda=J \equiv \operatorname{diag}\left(\lambda_{1}, \ldots, \lambda_{n}\right)
$$


We make the change of variables

$$
y=\Lambda z, \text { i.e., } \quad y_{j}=\sum_{m=1}^{n} \lambda_{m}^{j-1} z_{m},
$$

in (1.6) to obtain

$$
z^{\prime}=(J+G(t)) z
$$

where

$$
G(t)=\left(g_{j k}(t)\right)=\Lambda^{-1} Q(t) \Lambda .
$$

It is clear that $g_{j k}(t)$ is a linear combination of $q_{0}(t), \ldots, q_{n-1}(t)$ with coefficients depending on $j, k$ and $\lambda_{1}, \ldots, \lambda_{n}$. (The explicit formulas for $g_{j k}(t)$ will not be needed below, but a simple calculation shows that

$$
g_{j k}(t)=\sum_{m=0}^{n-1} q_{m}(t) \lambda_{k}^{m} / \pi_{j}
$$

where $\pi_{j}$ is defined by

$$
\pi_{j}=(-1)^{n} \prod_{h>j}\left(\lambda_{h}-\lambda_{j}\right)
$$

cf., e.g., [2, pp. 318-319].) Thus there exists a constant $M_{0}=M_{0}\left(\lambda_{1}, \ldots, \lambda_{n}\right)>0$ with the property that

$$
\left(\sum_{k=1}^{n}\left|g_{j k}(t)\right|^{2}\right)^{1 / 2} \leqq M_{0} q(t) \text { for } j=1, \ldots, n .
$$

(In fact, by (1.11), $M_{0}$ can be chosen to be $\|\Lambda\| / \pi$, where $\|\Lambda\|$ is the bound of the matrix $\Lambda$ as an operator from $R^{n}$ to $R^{n}$ and $\pi=\min \left(\left|\pi_{1}\right|, \ldots,\left|\pi_{n}\right|\right)$.)

Define the functions

$$
\sigma(t)=M_{0} \int_{0}^{t} q(s) e^{-c(t-s)} d s, \quad \tau(t)=M_{0} \int_{t}^{T} q(s) e^{-c(s-t)} d s
$$

and assume that the bound $\eta$ in (1.5) is so small that

$$
7 M_{0} \eta<1 .
$$

Then, for each $k$, [2, Lemma 4.2, p. 285] with $\varepsilon=0$ and $\psi(t)=M_{0} q(t)$ and its proof imply that (1.9) has a solution $z=\left(z_{k 1}(t), \ldots, z_{k n}(t)\right) \not \equiv 0$ satisfying, for $0 \leqq t<T$,

$$
\begin{array}{ll}
\left|z_{k j}(t)\right| \leqq 7 \sigma(t) z_{k k}(t) & \text { for } 1 \leqq j<k, \\
\left|z_{k j}(t)\right| \leqq 7 \tau(t) z_{k k}(t) & \text { for } k<j \leqq n ;
\end{array}
$$

cf. the Appendix below for a generalization. (Actually, the conditions of Lemma 4.2 in [2, p. 285] and its proof require that $\psi(t)=M_{0} q(t) \geqq 0$ be positive, but it is clear that the validity of Lemma 4.2 with $\psi(t)>0$ implies its validity for $\psi(t) \geqq 0$.) 
By (1.8), the corresponding solution $y=\left(y_{k 1}(t), \ldots, y_{k n}(t)\right)=\Lambda z$ of $(1.6)$ satisfies

$$
\left|y_{k j}(t)-z_{k k}(t) \lambda_{k}^{j-1}\right| \leqq 7 M_{0} \eta z_{k k}(t) \sum_{m \neq k}\left|\lambda_{m}\right|^{j-1}
$$

for $0 \leqq t<T$. The solution $y(t)$ of (1.6) corresponds to a solution $u=u_{k}(t)$ of (1.1) with the properties that if $e_{k j}(t)$ is defined by

$$
e_{k j}(t) z_{k k}(t)=u_{k}^{(j-1)}(t)-\lambda_{k}^{j-1} z_{k k}(t)
$$

then

$$
\left|e_{k j}(t)\right| \leqq C \eta, \quad \text { where } C=7 M_{0} \max _{j} \sum_{m=1}^{n}\left|\lambda_{m}\right|^{j-1}
$$

Let $1 \leqq m \leqq n$ and let $W_{m}(t)$ be the Wronskian determinant of the $m$ solutions $u_{1}(t), \ldots, u_{m}(t)$ of (1.1). Then

$$
W_{m}(t)=z_{11}(t) \cdots z_{m m}(t) \operatorname{det}\left(\lambda_{k}^{j-1}+e_{k j}\right)
$$

and, consequently, there exists a constant $K=K\left(\lambda_{1}, \ldots, \lambda_{n}\right)$ with the property that

$$
W_{n}(t)=z_{11}(t) \cdots z_{m m}(t)\left(\operatorname{det} \Lambda_{m}+\delta\right), \quad|\delta| \leqq K \eta(1+K \eta)^{n-1}
$$

and det $\Lambda_{m}=\Pi\left(\lambda_{k}-\lambda_{j}\right), 1 \leqq j<k \leqq m$. Hence, there exists an $\eta_{0}>0$ with the property that

$$
W_{m}(t) \neq 0 \quad \text { for } 0 \leqq t<T, m=1, \ldots, n \text { if } 0 \leqq \eta<\eta_{0}
$$

It follows from a theorem of Pólya [12, p. 317] that (1.1) is disconjugate on $0<t<T$.

In order to see that $(1.1)$ is disconjugate on $[0, T)$, that is, on every interval $[0, b), 0<b<T$, it is only necessary to extend the definition of $q_{k}(t)$ to an interval $[-\varepsilon, T)$, for a suitably small $\varepsilon=\varepsilon(b)>0$, and apply the statement already proved with $(0, T)$ replaced by $(-\varepsilon, b+\varepsilon)$.

COROllary 1.1. Let Assumption ( $\left.\mathrm{A}_{1}\right)$ hold. There exists a constant $\eta_{0}=$ $\eta_{0}\left(\lambda_{1}, \ldots, \lambda_{n}\right)>0$ with the property that if $q_{0}(t), \ldots, q_{n-1}(t)$ are continuous for $-\infty<t<\infty$ and $q(t)$ in (1.4) satisfies $q(t)<c \eta_{0}$ for all $t$, then (1.1) is disconjugate on $-\infty<t<\infty$.

This is a consequence of Theorem 1.1 which shows that the condition $q(t)<c \eta_{0}$ implies that (1.1) is disconjugate on every interval $[a, b),-\infty<a<b<\infty$.

COROllary 1.2. Let Assumption $\left(\mathrm{A}_{1}\right)$ hold. Let $q_{0}(t), \ldots, q_{n-1}(t)$ be continuous for $0 \leqq t<\infty$ and $q(t)$ be defined by (1.4). Let $u(t) \not \equiv$ be a solution of (1.1) and $N=N(T)$ the number of zeros of $u(t)$, counting multiplicities, on $0 \leqq t<T$. Then

$$
\eta_{0} N(T) \leqq(n-1) \int_{0}^{T} q(t) d t+\eta_{0}(n-1) .
$$


Proof. Let the nonnegative zeros (if any) of $u(t)$ be $0 \leqq t_{0} \leqq t_{1} \leqq \cdots$. Then

$$
\int_{s}^{t} q(\rho) d \rho \geqq \eta_{0} \quad \text { if } s=t_{j}, t=t_{j+n-1} .
$$

Thus if $t_{N-1}<T \leqq t_{N}$,

$$
\int_{0}^{T} q(\rho) d \rho \geqq \eta_{0}[(N-1) /(n-1)] \geqq \eta_{0}\{(N-1) /(n-1)-(n-2) /(n-1)\} .
$$

This gives (1.15).

2. Coincident real roots. We now consider the case when Assumption $\left(A_{1}\right)$ does not hold and, in fact, the extreme opposite case, $\lambda_{1}=\cdots=\lambda_{n}$, holds. We suppose that the value of $\lambda_{1}=\cdots=\lambda_{n}$ is 0 ; cf. the Remark at the end of this section.

THEOREM 2.1. There exists a number $\eta_{1}>0$, independent of $T$, with the following property: In the differential equation

$$
u^{(n)}=q_{n-1}(t) u^{(n-1)}+\cdots+q_{0}(t) u,
$$

let $q_{0}(t), \ldots, q_{n-1}(t)$ be continuous for $0 \leqq t<T(\leqq \infty)$ [or for $0 \leqq t \leqq T(<\infty)$ ] and such that

$$
q(t)=\left(\sum_{k=0}^{n-1}\left|q_{k}(t) t^{n-k-1}\right|^{2}\right)^{1 / 2} \geqq 0
$$

satisfies

$$
t^{-1} \int_{0}^{t} s q(s) d s, \quad t \int_{t}^{T} s^{-1} q(s) d s \leqq \eta<\eta_{1}
$$

(in particular, let

$$
\int_{0}^{T} q(t) d t<\eta_{1} \quad \text { or } \quad t q(t)<\eta_{1}
$$

hold), then (2.1) is disconjugate on $0 \leqq t<T(\leqq \infty)$ [or on $0 \leqq t \leqq T(<\infty)$ ].

If $n=2$ and $q_{1}(t) \equiv 0$, then the first criterion in (2.4) is known with $\eta_{1}=1$ (cf. [4, Theorem 5.1, p. 345] with $m(t)=t-a)$ and the second is an analogue of A. Kneser's criterion $t^{2} q_{0}(t) \leqq 1 / 4$. If $n \geqq 2, q_{1}(t) \equiv \cdots \equiv q_{n-1}(t) \equiv 0$ and $q_{0}(t)$ is of constant sign and monotone, then the first criterion in (2.4) has been proved by Nehari [12]. (For a related result of de la Vallée Poussin and generalizations, see Levin [9], [10], Nehari [11], and Hukuhara [7].) According to a theorem of Dunkel (cf. [2, Theorem 17.1, p. 315]), the convergence of the integral in (2.4) for $T=\infty$ implies asymptotic formulae for the solutions of (2.1) for large $t$. But these formulae do not imply a disconjugacy criterion for (2.1) on $t \geqq 0$.

Proof. Under the change of independent variables

$$
s=-\log t \text { or } t=e^{-s}
$$


the interval $0<t<T$ is changed to $-\log T<s<\infty$. Note that $u^{\prime}=-e^{s} D u$, where $D=d / d s$. Thus

$$
u^{(k)}=(-t)^{-k} D(D+1) \cdots(D+k-1) u
$$

Write this relation as

$$
u^{(k)}=(-t)^{-k} \sum_{j=1}^{k} \beta_{k j} D^{j}, \quad \text { where } \beta_{k k}=1,
$$

so that

$$
\sum_{j=1}^{k} \beta_{k j} \lambda^{j}=\lambda(\lambda+1) \cdots(\lambda+k-1)
$$

Thus (2.1) becomes

$$
D^{n} u+\sum_{j=1}^{n-1} \beta_{n j} D^{j} u=Q_{n-1}(s) D^{n-1} u+\cdots+Q_{0}(s) u,
$$

where $t=e^{-s}, Q_{0}(s)=(-t)^{n} q_{0}(t)$, and

$$
Q_{j}(s)=\sum_{k=j}^{n-1}(-t)^{n-k} q_{k}(t) \beta_{k j} \quad \text { for } j=1, \ldots, n-1 .
$$

Hence, there is a constant $C=C(n)$ such that

$$
Q(s)=\left(\sum_{j=0}^{n-1}\left|Q_{j}(s)\right|^{2}\right)^{1 / 2} \leqq C q(t) t
$$

where $q(t)$ is defined by (2.2).

It is clear from (2.6) that the roots of the characteristic polynomial belonging to the left side of (2.7) are $0,-1, \ldots,-(n-1)$. In view of (2.9), Theorem 2.1 follows from Theorem 1.1 after an obvious change of integration variables.

Theorem 2.1 will be combined with the method of [3] for the case $n=2, q_{1}(t) \equiv 0$ (cf. [2, pp. 346-347]) to obtain an analogue of Corollary 1.2.

COROllary 2.1. There exist positive constants $M_{0}, \ldots, M_{n-1}$ with the following property: Let $q_{0}(t), \ldots, q_{n-1}(t)$ be continuous for $t \geqq 0, u(t) \equiv 0$ a solution of $(2.1)$, and $N=N(T)$ the number of zeros of $u(t)$ on $0 \leqq t<T(<\infty)$, counting multiplicities. Then $N \leqq n-1$ or $N$ satisfies the inequality

$$
\sum_{k=0}^{n-1} M_{k}\{N /(n-1)-1\}^{k-n}\left\{T^{n-1} \int_{0}^{T}\left|q_{k}(t)\right|^{n /(n-k)} d t\right\}^{1-k / n}>1 .
$$

Since $k-n<0,(2.10)$ can be used to estimate $N=N(T)$ from above. An admissible choice for $M_{0}, \ldots, M_{n-1}$ is given by $M_{k}=2^{k-n}, k=0, \ldots, n-1$. This follows from the proof of Corollary 2.1 and a result of Nehari [11]. (Another choice of the constants may be given in the paper by Hukuhara [7] which is not available to me; the pertinent (last) result stated in the review in the Mathematical Reviews does 
not seem correct.) Still another choice is, e.g., $M_{0}=(n-2)^{n-2} /(n-1)^{n-1}(n-1)$ ! and $M_{k}=1 /(n-k-1)$ ! for $k=1, \ldots, n-1$; cf. the argument in [2, Exercise 5.3, p. 570]. See also Levin [9], [10].

Proof. Let $N>n-1$ and $(0 \leqq) t_{0} \leqq t_{1} \leqq \cdots$ be the nonnegative zeros of $u(t)$; so that $t_{N-1}<T \leqq t_{N}$. By Theorem 2.1, there exist positive constants $M_{0}, \ldots, M_{n-1}$ such that

$$
\sum_{k=0}^{n-1} M_{k}(t-s)^{-k} \int_{s}^{t}\left|q_{k}\right| d \rho>(t-s)^{1-n} \text { if } s=t_{j}, t=t_{j+n-1}
$$

e.g., by (2.2) and (2.4), any set of constants $M_{k}>1 / \eta_{1}$ for $k=0, \ldots, n-1$ is admissible. By [11], one can choose $M_{k}=2^{-k}$ for $k=0, \ldots, n-1$.

Letting $s=t_{j}, t=t_{j+n-1}$ and adding for $j=0, \ldots, m$ gives

$$
\sum_{k=0}^{n-1} M_{k} \sum_{j=0}^{m}(t-s)^{-k} \int_{s}^{t}\left|q_{k}\right| d \rho>\sum_{j=0}^{m}(t-s)^{1-n} .
$$

Note that if $1<\alpha<\infty$,

$$
(t-s)^{-k} \int_{s}^{t}\left|q_{k}\right| d \rho \leqq(t-s)^{1-k-1 / \alpha}\left(\int_{s}^{t}\left|q_{k}\right|^{\alpha} d \rho\right)^{1 / \alpha}
$$

Thus, if $1 / \alpha+1 / \beta=1$,

$$
\sum_{j=0}^{m}(t-s)^{-k} \int_{s}^{t}\left|q_{k}\right| d \rho \leqq\left(\sum_{j=0}^{m}(t-s)^{\beta(1-k-1 / \alpha)}\right)^{1 / \beta}\left(\int_{t_{0}}^{t_{m(n-1)}}\left|q_{k}\right|^{\alpha} d \rho\right)^{1 / \alpha} .
$$

For $1 \leqq k \leqq n-1$, choose $\alpha=n /(n-k)$ and $\beta=n / k$, so that $\beta(1-k-1 / \alpha)=1-n$ and the last product is

$$
\left(\sum_{j=0}^{m}(t-s)^{1-n}\right)^{k / n}\left(\int_{t_{0}}^{t_{m(n-1)}}\left|q_{k}\right|^{n /(n-k)} d \rho\right)^{1-k / n}
$$

Thus, by (2.11),

$$
\sum_{k=0}^{n-1} M_{k}\left(\sum_{j=0}^{m}(t-s)^{1-n}\right)^{-1+k / n}\left(\int_{t_{0}}^{t_{m(n-1)}}\left|q_{k}\right|^{n /(n-k)} d \rho\right)^{1-k / n}>1 .
$$

From the inequality for the harmonic mean,

$$
\left[\sum_{j=0}^{m}(t-s)^{-(n-1)}\right]^{-1 /(n-1)} \leqq(m+1)^{-n /(n-1)} \sum_{j=0}^{m}(t-s) .
$$

The last sum is $t_{m(n-1)}-t_{0}<T$ if $t_{m(n-1)} \leqq t_{N-1}<T$. Thus we obtain

$$
\sum_{k=0}^{n-1} M_{k}\left[(m+1)^{-n} T^{n-1} \int_{0}^{T}\left|q_{k}\right|^{n /(n-k)} d s\right]^{1-k / n}>1 .
$$

If we choose $m+1=[(N-1) /(n-1)] \geqq(N-1) /(n-1)-(n-2) /(n-1)$, then $m+1$ $\geqq N /(n-1)-1$. This, together with the last inequality, gives $(2.10)$ and proves Corollary 2.1 . 
REMARK. The analogues of Theorem 2.1 and its corollary in which (2.1) is replaced by

$$
(d / d t-\lambda)^{n} u=q_{n-1}(t) u^{(n-1)}+\cdots+q_{0}(t) u
$$

and (2.2) by

$$
q(t)=\left(\left|q_{0}(t) t^{n-1}\right|^{2}+\sum_{k=1}^{n-1}\left|q_{k}(t) t^{n-k-1}\left(1+|\lambda t|^{k}\right)\right|^{2}\right)^{1 / 2},
$$

are valid (and the corresponding number $\eta_{1}$ does not depend on $\lambda$ ).

In fact, the change of dependent variables

$$
v=e^{-\lambda t} u
$$

transforms (2.12) into an equation of the form

where

$$
v^{(n)}=\rho_{n-1}(t) v^{(n-1)}+\cdots+\rho_{0}(t) v
$$

$$
\rho_{j}(t)=\sum_{k=j}^{n-1} C_{k j} \lambda^{k-j} q_{k}(t), \quad \text { where } C_{k j}=k ! / j !(k-j) ! .
$$

Hence, (2.13) satisfies

$$
\sum_{k=0}^{n-1}\left|\rho_{k}(t) t^{n-k-1}\right|^{2} \leqq C^{2}|q(t)|^{2}
$$

for a suitable constant $C$, independent of $\lambda$.

3. Arbitrary real roots. We now consider the differential equation

$$
u^{(n)}+a_{n-1} u^{(n-1)}+\cdots+a_{0} u=q_{n-1}(t) u^{(n-1)}+\cdots+q_{0}(t) u,
$$

when the following holds:

Assumption $\left(\mathrm{A}_{2}\right)$. Let $a_{0}, \ldots, a_{n-1}$ be real numbers such that the polynomial

$$
\lambda^{n}+a_{n-1} \lambda^{n-1}+\cdots+a_{0}=0
$$

has only real roots, say, $\lambda(1), \ldots, \lambda(g)$, with the respective multiplicities $h(1), \ldots, h(g)$; so that $h(j) \geqq 1$ and $h(1)+\cdots+h(g)=n$. Let

$$
h_{*}=\max (h(1), \ldots, h(g)) \text {. }
$$

THEOREM 3.1. Let Assumption $\left(\mathrm{A}_{2}\right)$ hold. Then there exist numbers $C \geqq 1$ and $\eta_{2}>0$, depending on $a_{0}, \ldots, a_{n-1}$ but independent of $T$, with the following property: If $q_{0}(t), \ldots, q_{n-1}(t)$ are continuous on $0 \leqq t<T(\leqq \infty)$ [or on $0 \leqq t \leqq T(<\infty)$ ] and

$$
q(t)=(1+t)^{n_{*}-1} \sum_{k=0}^{n-1}\left|q_{k}(t)\right|
$$

satisfies

$$
\int_{0}^{t} q(s)[(s+C) /(t+C)] d s, \int_{t}^{T} q(s)[(t+C) /(s+C)] d s \leqq \eta<\eta_{2}
$$


or, in particular,

$$
\int_{0}^{T} q(t) d t<\eta_{2} \text { or }(t+C) q(t)<\eta_{2}
$$

then (3.1) is disconjugate on $0 \leqq t<T(\leqq \infty)$ [or on $0 \leqq t \leqq T(<\infty)$ ].

The proof depends on a method of Hartman and Wintner [5] for the asymptotic integration of linear systems with nearly constant coefficients. This method involves a change of variables which enables us to obtain rather precise information on the asymptotic behavior of each component of the solution vector.

Proof. We shall deal only with disconjugacy for $0<t<T$. The passage to $0 \leqq t<T$ can be made as in the proof of Theorem 1.1.

(a) Let $C=C\left(a_{0}, \ldots, a_{n-1}\right) \geqq 1$ be a number to be fixed below and introduce the abbreviation

$$
s=t+C \geqq 1 \quad \text { if } \quad 0 \leqq t<T .
$$

Assume, that for each $j$, there exist $h(j)$ solutions $u=u_{j 1}(t), \ldots, u_{j h(j)}(t)$ of $(3.1)$ with the properties that $v_{j k}(t)=u_{j k}(t) e^{-\lambda(j) s}$ and its derivatives satisfy

$$
\begin{array}{rlrl}
\left|D^{i-1} v_{j k}(t)-w_{\kappa}^{j}(t) s^{x-i} /(\kappa-1) !\right| & \leqq \gamma \eta\left|w_{\kappa}^{j}(t)\right| s^{x-i} & & \text { for } i=1, \ldots, \kappa, \\
\left|D^{i-1} v_{j k}(t)\right| \leqq \gamma \eta\left|w_{x}^{j}(t)\right| s^{x-i} & & \text { for } i=\kappa+1, \ldots, h(j), \\
\left|D^{i-1} v_{j k}(t)\right| \leqq \gamma \eta\left|w_{x}^{j}(t)\right| s^{x-h_{*}} & & \text { for } h(j)<i \leqq n,
\end{array}
$$

for $0 \leqq t<T$, where $w_{x}^{j}(t)$ is a continuous nonvanishing function; $\gamma$ denotes a constant, not always the same, depending only on $a_{0}, \ldots, a_{n-1}$; and $D=d / d t$.

It will now be shown that if $\eta>0$ is sufficiently small and the $n$ solutions $u_{11}, \ldots$, $u_{1 h(1)}, u_{21}, \ldots, u_{g h(g)}$ are numbered as $u_{1}, \ldots, u_{n}$, then the Wronskian determinant

$$
W_{m}(t)=\operatorname{det}\left(D^{i-1} u_{j}\right), \quad \text { where } i, j=1, \ldots, m(\leqq n),
$$

does not vanish for $0 \leqq t<T$.

Let $1 \leqq m \leqq n$; say, $m=h(1)+\cdots+h(\mu-1)+\nu$, where $1 \leqq \nu \leqq h(\mu)$. Let $h^{\prime}(j)=h(j)$ or $\nu$ according as $j \leqq \mu-1$ or $j=\mu$. The entry $D^{i-1} u_{j x}$ in $W_{m}(t)$ is

$$
D^{i-1} u_{j \kappa}=D^{i-1} e^{\lambda(j) s} v_{j \kappa}=e^{\lambda(j) s} \sum_{r=1}^{i} C_{i-1, r-1} \lambda^{i-r}(j) D^{r-1} v_{j \kappa}
$$

If $i \leqq h(j)$, let $e_{i h x}(t)=0$. If $i>h(j)$, let

$$
e_{i j x}(t)=\sum_{r=h(j)+1}^{i} C_{i-1, r-1} \lambda^{i-r}(j) D^{r-1} v_{j \kappa},
$$

so that, by (3.8),

$$
\left|e_{i j \kappa}(t)\right| \leqq \gamma\left|w_{\kappa}^{j}(t)\right| \eta s^{x-h_{\bullet}} \quad \text { for } 0 \leqq t<T ; \kappa=1, \ldots, h(j) ; i=1, \ldots, n
$$


The relations (3.9), (3.10) give

$$
D^{i-1} u_{j k}=e^{\lambda(j) s}\left[f_{i j k}(t)+e_{i j k}(t)\right]
$$

where

$$
f_{i j \kappa}=\sum_{r=1}^{\min (i, h(j))} C_{i-1, r-1} \lambda^{i-r}(j) D^{r-1} v_{j \kappa} .
$$

The $m \times m$ matrix $\left(f_{i j k}(t)\right)$, corresponding to $\left(D^{i-1} u_{j}\right), i, j=1, \ldots, m$, can be written as the product $\Lambda_{(m)} W^{m}(t)$ of two $m \times m$ matrices. The matrix $\Lambda_{(m)}$ is a constant matrix which is the Wronskian determinant at $t=0$ of the first $m$ functions of $\omega_{11}, \ldots, \omega_{1 h(1)}, \omega_{21}, \ldots, \omega_{g h(g)}$, where $\omega_{j k}=e^{\lambda(j) t} t^{k-1} /(k-1)$ !. By (3.9),

$$
D^{i-1} \omega_{j r}(0)=\sum_{k} C_{i-1, k-1} \lambda^{i-k}(j)\left[t^{r-k} /(r-k) !\right]_{t=0},
$$

and the sum is over the range $1 \leqq k \leqq \min (i, r)$; so that

$$
D^{i-1} \omega_{j r}(0)=C_{i-1, r-1} \text { or } 0 \text {, according as } r \leqq i \text { or } r>i \text {. }
$$

Choose $W^{m}(t)$ to be the matrix

$$
W^{m}(t)=\operatorname{diag}\left(W_{(1)}(t), \ldots, W_{(\mu)}(t)\right),
$$

where $W_{(j)}(t)$ is an $h^{\prime}(j) \times h^{\prime}(j)$ matrix,

$$
W_{(j)}(t)=\left(D^{i-i} v_{j \kappa}\right) \text { for } i, \kappa=1, \ldots, h^{\prime}(j) .
$$

Thus, we have $\left(f_{i j x}(t)\right)=\Lambda_{(m)} W^{m}(t)$.

The matrix $\Lambda_{(m)}$ is nonsingular, since its determinant is the Wronskian determinant of $m$ linearly independent solutions of an $m$ th order linear equation $u^{(m)}+\cdots=0$ with constant coefficients. Thus, by (3.12), the matrix $\left(D^{i-1} u_{j}\right)$ can be written

$$
\left(D^{i-1} u_{j x}\right)=\Lambda_{(m)}\left[W^{m}(t)+\Lambda_{(m)}^{-1}\left(e_{i j k}\right)\right] D_{0}
$$

where $D_{0}=\operatorname{diag}\left(e^{\lambda(1) s}, \ldots, e^{\lambda(\mu) s}\right)$ in which $e^{\lambda(j) s}$ occurs $h^{\prime}(j)$ times. Thus, the assertion $W_{m}(t) \neq 0$ is equivalent to

$$
\operatorname{det} W^{(m)}(t) \neq 0, \text { where } W^{(m)}=W^{m}(t)+\Lambda_{(m)}^{-1}\left(e_{i j x}\right) .
$$

From (3.11), the absolute values of the elements in the columns of $\Lambda_{(m)}^{-1}\left(e_{i j k}\right)$ corresponding to $u_{j x}$ are majorized by $\gamma\left|w_{x}^{j}(t)\right| \eta s^{x-h_{*}}$. Divide the corresponding column of $W^{(m)}(t)$ by $s^{x-1}\left|w_{x}^{j}(t)\right|$ and multiply the $[h(1)+\cdots+h(j-1)+i]$ th row by $s^{i-1}$. In the resulting matrix, the elements which are not in the blocks corresponding to $W_{(1)}, \ldots, W_{(\mu)}$ (cf. (3.14)) are majorized by $\gamma \eta s^{i-h_{*}}$, where $i-h_{*} \leqq h(j)-h_{*} \leqq 0$. Since $s \geqq 1$ for $t \geqq 0, \gamma \eta s^{i-h_{*}} \leqq \gamma \eta$. By the same argument, the elements below the diagonals on the blocks $W_{(1)}, \ldots, W_{(\mu)}$ are majorized by $\gamma \eta s^{i-x} \leqq \gamma \eta$ for $i \leqq \kappa$. The elements on and above the diagonal become $1 /(i-\kappa)$ ! +error, where |error $\mid \leqq \gamma \eta$; in particular, the diagonal elements differ from 1 by at most $\gamma \eta$. Thus, it is clear that if $\eta_{2}$ is sufficiently small and $\eta<\eta_{2}$, then (3.15) holds. 
(b) In order to complete the proof of Theorem 3.1, it is necessary to prove the existence of the solutions $u=u_{j k}(t)$ assumed in part (a). Let $j$ be fixed, $1 \leqq j \leqq g$. For the sake of definiteness let $j=1, \lambda=\lambda(1), h=h(1)$ and put $\mu(j)=\lambda(j)-\lambda$ for $j=1, \ldots, g$. In particular $\mu(1)=0$. In (3.1), introduce the new dependent variable

$$
v=e^{-\lambda t} u \text {. }
$$

Then (3.1) takes the form

$$
v^{(n)}+b_{n-1} v^{(n-1)}+\cdots+b_{h} v^{(h)}=\sum_{i=0}^{n-1} \rho_{i}(t) v^{(i)} .
$$

Here $b_{h}, \ldots, b_{n-1}$ are constants such that the roots of

$$
\tau^{n}+b_{n-1} \tau^{n-1}+\cdots+b_{h} \tau^{h}=0
$$

are $0=\mu(1), \mu(2), \ldots, \mu(g)$ with the respective multiplicities $h(1)^{\prime}, \ldots, h(g)$. Also

$$
\rho_{i}(t)=\sum_{k=i}^{n-1} C_{k, i} \lambda^{k-i} q_{k}(t), \quad \text { where } C_{k, i}=k ! / i !(k-i) !
$$

Write (3.17) as a first order system

$$
y^{\prime}=B y+P(t) y
$$

for the vector $y=\left(v, v^{\prime}, \ldots, v^{(n-1)}\right)$; cf. (1.1) and (1.6).

For a given $j$, let $J_{j}$ denote the $h(j) \times h(j)$ Jordan matrix with the diagonal elements $\mu(j)$ and, if $h(j)>1$, superdiagonal elements 1 . The first $h$ columns of $B$ constitute an $h \times n$ matrix with $J_{1}$ in the upper portion and the zero matrix $0_{n \times(n-h)}$ below. Thus, there exists a nonsingular constant matrix of the form

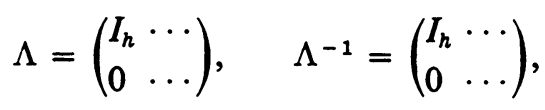

such that $I_{h}$ is the unit $h \times h$ matrix and

$$
\Lambda^{-1} B \Lambda=J \equiv \operatorname{diag}\left(J_{1}, \ldots, J_{\vartheta}\right) .
$$

The linear change of variables

$$
y=\Lambda z
$$

sends (3.19) into

$$
z^{\prime}=J z+\Lambda^{-1} P(t) \Lambda z
$$

Write $z=\left(z^{1}, \ldots, z^{g}\right)$, where $z^{j}=\left(z_{1}^{j}, \ldots, z_{h(g)}^{j}\right)$ is a vector of dimension $h(g)$, and (3.23) as

$$
z^{j^{\prime}}=J_{j} z^{j}+\sum_{k=1}^{g} P^{j k}(t) z^{k} \quad \text { for } j=1, \ldots, g
$$

where $P^{j k}(t)$ is an $h(j) \times h(k)$ matrix. 
Since $P(t)$ is an $n \times n$ matrix with 0 entries except for the last row $\left(-\rho_{0}(t), \ldots\right.$, $\left.-\rho_{n-1}(t)\right)$, it follows from (3.20), (2.23) that the $i$ th component of $P^{j 1}(t) z^{1}$ is of the form

$$
\left(P^{j 1}(t) z^{1}\right)_{i}=\alpha_{i}^{1} \sum_{m=0}^{n-1} \rho_{m}(t) z_{m+1}^{1},
$$

where $\left(-\alpha_{1}^{1}, \ldots,-\alpha_{h(g)}^{g}\right)$ is the last column of $\Lambda^{-1}$. Furthermore the elements of $P^{j k}(t)$ are linear combinations of $\rho_{0}(t), \ldots, \rho_{n-1}(t)$ with constant coefficients.

Let $C \geqq 1, \varepsilon>0$ be constants to be specified below and introduce the abbreviation $s=t+C$, as in (a) above. (Note that $t$ is still the independent variable.) Make the linear change of variables $z \rightarrow w$ :

$$
z^{1}=\left(\exp J_{1} s\right) w^{1} \text { and } z_{i}^{j}=\varepsilon^{h(j)-i} w_{i}^{j}
$$

for $j=2, \ldots, g$, to obtain

$$
w^{1^{\prime}}=\sum_{k=1}^{g} Q^{1 k}(t) w^{k} \quad \text { and } \quad w^{j^{\prime}}=J_{j \varepsilon} w^{j}+\sum_{k=1}^{g} Q^{j k}(t) w^{k}
$$

where $J_{j \varepsilon}$ is obtained by replacing the superdiagonal elements 1 in $J_{j}$ by $\varepsilon$. If (3.26) is written as

$$
z=Q_{0}(t) w, Q_{0}(t)=\operatorname{diag}\left(\exp J_{1} s, D_{1}\right),
$$

where $D_{1}=D_{1}(\varepsilon)$ is a diagonal matrix, then (3.27) is

$$
w^{\prime}=\operatorname{diag}\left(0, J_{2 \varepsilon}, \ldots, J_{g \varepsilon}\right) w+Q_{0}^{-1} \Lambda^{-1} P \Lambda Q_{0} w .
$$

From this, we can write (3.27) in detail as

$$
w_{i}^{1^{\prime}}=\left(\sum_{r=i}^{h} c_{i r} s^{r-i}\right) \sum_{k=1}^{h} \sum_{m=0}^{k-1} d_{k m} \rho_{m}(t) s^{k-1-m} w_{k}^{1}+\sum_{l=2}^{g} \sum_{m=1}^{n(1)} \sum_{r=i}^{h} L_{1 i, l m r}(t) s^{r-i} w_{m}^{l},
$$

$i=1, \ldots, h$, and

(3.29) $w_{i}^{j^{\prime}}=\mu_{j} w_{i}^{j}+\varepsilon_{j i} w_{i+1}^{j}+\sum_{k=1}^{n} \sum_{m=0}^{k-1} d_{j i, k m} \rho_{m}(t) s^{k-1-m} w_{k}^{1}+\sum_{l=2}^{g} \sum_{m=1}^{h(1)} L_{j i, l m}(t) w_{m}^{l}$,

for $j=2, \ldots, g$ and $i=1, \ldots, h(j)$, where $c_{i r}, d_{k m}, d_{j i, k m}$ are constants; $L_{1 i, l m k}(t)$ and $L_{j i, l m}(t)$ are linear functions of $\rho_{0}(t), \ldots, \rho_{n-1}(t)$ with constant coefficients; and $\varepsilon_{j i}=\varepsilon$ or 0 according as $i<h(j)$ or $i=h(j)$.

Let $1 \leqq \kappa \leqq h$. Make the last change of variables $w \rightarrow x$ :

$$
x_{i}^{1}=s^{i-x} w_{i}^{1} \text { and } x_{i}^{j}=s^{h_{*}-x} w_{i}^{j}, \quad j>1 .
$$

Then (3.28)-(3.29) becomes

$$
\begin{aligned}
x_{i}^{1^{\prime}}=(i-\kappa) s^{-1} x_{i}^{1}+\sum \sum \sum c_{i r} d_{k m} \rho_{m}(t) s^{r-m-1} x_{k}^{i}+\sum \sum \sum L_{1 i, l m r}(t) s^{r-h_{*}} x_{m}^{l}, \\
\begin{aligned}
x_{i}^{j^{\prime}}=\left[\mu(j)+\left(h_{*}-\kappa\right) s^{-1}\right] x_{i}^{i}+ & \varepsilon_{j i} x_{i+1}^{j} \\
& +\sum \sum d_{j i, k m} \rho_{m}(t) s^{h_{*}-m-1}+\sum \sum L_{j i, l m}(t) x_{m}^{l},
\end{aligned}
\end{aligned}
$$

where the ranges for the sums are the same as in (3.28), (3.29), respectively. 
Choose $C \geqq 1$ so large and $\varepsilon>0$ so small that

$$
[\operatorname{sgn} \mu(j)] J_{j \varepsilon} x^{j} \cdot x^{j}-\left(h_{*}-\kappa\right) s^{-1}\left|x^{j}\right|^{2} \geqq s^{-1}\left|x^{j}\right|^{2} \quad \text { for } j>1 .
$$

Since $s=t+C \geqq 1$ when $t \geqq 0$, it is clear that there exists a constant

$$
C_{1}=C_{1}\left(a_{0}, \ldots, a_{n-1}\right)
$$

such that if

$$
\psi(t)=C_{1} \sum_{m=0}^{n-1}\left|\rho_{m}(t)\right| s^{h_{*}-1},
$$

then

$$
|\{\cdots\}| \leqq \psi(t)|x| \quad \text { if }|x|^{2}=\sum_{j=1}^{g}\left|x^{j}\right|^{2}=\sum_{j=1}^{g} \sum_{k=1}^{h(j)}\left|x_{k}^{j}\right|^{2}
$$

and $\{\cdots\}$ denotes either the sum of the two triple sums in (3.31) or the sum of the two double sums in (3.32). Thus, (3.31)-(3.32) imply that

$$
\begin{aligned}
\sum_{i=1}^{\kappa-1} x_{i}^{1} x_{i}^{1^{\prime}} & \leqq-s^{-1} \sum_{i=1}^{\kappa-1}\left|x_{i}^{1}\right|^{2}+\psi(t)|x|\left(\sum_{i=1}^{\kappa-1}\left|x_{i}^{1}\right|^{2}\right)^{1 / 2}, \\
x^{j} \cdot x^{j^{\prime}} & \leqq-s^{-1}\left|x^{j}\right|^{2}+\psi(t)|x| \cdot\left|x^{j}\right| \quad \text { if } \mu(j)<0, \\
\left|x_{\kappa}^{1} x_{\kappa}^{1^{\prime}}\right| & \leqq \psi(t)|x| \cdot\left|x_{\kappa}^{1}\right|, \\
\sum_{i=\kappa+1}^{n} x_{i}^{1} x_{i}^{1^{\prime}} & \geqq s^{-1} \sum_{i=\kappa+1}^{n}\left|x_{i}^{1}\right|^{2}-\psi(t)|x|\left(\sum_{i=\kappa+1}^{n}\left|x_{i}^{1}\right|^{2}\right)^{1 / 2}, \\
x^{j} \cdot x^{j^{\prime}} & \geqq s^{-1}\left|x^{j}\right|^{2}-\psi(t)|x| \cdot\left|x^{j}\right| \quad \text { if } \mu(j)>0 .
\end{aligned}
$$

We can now apply Theorem (*) of the Appendix below (with $y^{x}$ corresponding to the 1-dimensional $x_{\kappa}^{1}$ with $\alpha^{\kappa}=\alpha_{\kappa}=0$; the vectors $y^{1}, \ldots, y^{\kappa-1}$ correspond to $y^{1}=\left(x_{1}^{1}, \ldots, x_{x-1}^{1}\right)$ and the $x^{j}$ for which $\mu(j)<0$ with the respective $\alpha_{1}=\alpha_{2}=\cdots$ $=\alpha_{x-1}=-s^{-1}$; the vectors $y^{x+1}, \ldots, y^{n}$ correspond to $y^{x+1}=\left(x_{\kappa+1}^{1}, \ldots, x_{h}^{1}\right)$ and the $x^{j}$ for which $\mu(j)>0$ with the respective $\alpha_{x+1}=\alpha_{x+2}=\cdots=s^{-1}$.

Thus, we have to examine the functions

$$
\sigma_{1}=\int_{0}^{t} \psi(\rho)(\rho+C) /(t+C) d \rho, \quad \sigma_{x+1}=\int_{t}^{T} \psi(\rho)(t+C) /(\rho+C) d \rho .
$$

According to Theorem $(*)$, if $\sigma_{1}$ and $\sigma_{x+1}$ exist and have a sufficiently small bound $\eta$, then (3.31)-(3.32) has a solution $x(t) \not \equiv 0$ such that

$$
\left|x_{i}^{1}\right| \leqq \gamma \eta\left|x_{\kappa}^{1}\right| \quad \text { for } i \neq \kappa, \quad\left|x^{j}\right| \leqq \gamma \eta\left|x_{\kappa}^{1}\right| \quad \text { for } j \neq 1,
$$

$0 \leqq t<T$, where $\gamma$ denotes a constant independent of $\eta$, but not always the same. In view of (3.30), this means that (3.28)-(3.29) has a solution $w(t) \neq 0$ such that

$$
\left|w_{i}^{1}(t)\right| \leqq \gamma \eta s^{x-i}\left|w_{x}^{1}(t)\right| \text { and }\left|w^{j}(t)\right| \leqq \gamma \eta s^{x-h_{\bullet}}\left|w_{x}^{1}(t)\right|
$$


for $i \neq \kappa, j \neq 1$, respectively. By (3.26), (3.24) has a solution $z(t) \not \equiv 0$ satisfying

$$
\begin{array}{rlrl}
\left|z_{i}^{1}(t)-w_{\kappa}^{1}(t) s^{\kappa-i} /(\kappa-i) !\right| & \leqq \eta \eta\left|w_{\kappa}^{1}(t)\right| s^{\kappa-i} & & \text { for } i=1, \ldots, \kappa, \\
\left|z_{i}^{1}(t)\right| & \leqq \eta \eta\left|w_{\kappa}^{1}(t)\right| s^{\kappa-i} & & \text { for } i=\kappa+1, \ldots, h, \\
\left|z^{j}(t)\right| \leqq \gamma \eta\left|w_{\kappa}^{1}(t)\right| s^{\kappa-h_{\bullet}} & & \text { for } j=2, \ldots, g .
\end{array}
$$

Finally, by (3.20) and (3.22), (3.19) has a solution $y=\left(v, v^{\prime}, \ldots, v^{(n-1)}\right)$ satisfying (3.8) if $j=1$ and $v=v_{j x}$. This completes the proof of Theorem 3.1.

4. Nonlinear interpolation problems. The results and methods of Lasota and Opial [8], combined with those above, make it possible to treat nonlinear boundary value or interpolation problems associated with certain nonlinear differential equations

$$
\begin{gathered}
u^{(n)}+a_{n-1} u^{(n-1)}+\cdots+a_{0} u=f\left(t, u, u^{\prime}, \ldots, u^{(n-1)}\right), \\
u^{i-1}\left(t_{k}\right)=c_{i k} \text { for } i=1, \ldots, m(k) \text { and } k=1, \ldots, \nu, \\
(0=) t_{1}<\cdots<t_{\nu}(=T) \text { and } m(1)+\cdots+m(\nu)=n .
\end{gathered}
$$

AsSumption $\left(\mathrm{B}_{1}\right)$. Let $a_{0}, \ldots, a_{n-1}$ be real numbers satisfying Assumption $\left(\mathrm{A}_{1}\right)$ of Theorem 1.1, or the assumption $a_{0}=\cdots=a_{n-1}=0$ of Theorem 2.1, or Assumption $\left(\mathrm{A}_{2}\right)$ of Theorem 3.1. Correspondingly, let $0<T<\infty$ and let $q_{0}(t), \ldots, q_{n-1}(t)$ be nonnegative, continuous functions on $0 \leqq t \leqq T$ such that $q(t)$ defined in (1.4) satisfies (1.5), or $q(t)$ defined in (2.2) satisfies (2.3), or $q(t)$ defined by (3.4) satisfies (3.5).

Assumption $\left(\mathrm{B}_{2}\right)$. Let $f\left(t, y_{0}, \ldots, y_{n-1}\right)$ be a continuous function for $0 \leqq t \leqq T$ and arbitrary $\left(y_{0}, \ldots, y_{n-1}\right)$ satisfying

$$
\left|f\left(t, y_{0}, \ldots, y_{n-1}\right)\right| \leqq \sum_{k=0}^{n-1} q_{k}(t)\left|y_{k}\right| .
$$

THEOREM 4.1. Let $a_{0}, \ldots, a_{n-1}$ and $q_{0}(t), \ldots, q_{n-1}(t)$ satisfy Assumption $\left(\mathrm{B}_{1}\right)$ and $f\left(t, y_{0}, \ldots, y_{n-1}\right)$ satisfy Assumption $\left(\mathrm{B}_{2}\right)$. Then the interpolation problem (4.1)(4.3), in which $c_{11}, \ldots, c_{m(v) v}$ are arbitrary constants, has at least one solution. If, in addition, $f\left(t, y_{0}, \ldots, y_{n-1}\right)$ satisfies

$$
\left|f\left(t, y_{0}, \ldots, y_{n-1}\right)-f\left(t, z_{0}, \ldots, z_{n-1}\right)\right| \leqq \sum_{k=0}^{n-1} q_{k}(t)\left|y_{k}-z_{k}\right|,
$$

then the solution of (4.1)-(4.3) is unique.

Proof. If $\nu=n$ and $m(1)=\cdots=m(n)=1$, then this theorem follows from [8]. In fact, since $T$ is finite and the main conditions in (1.5), (2.3), (3.5) involve a strict inequality, it is clear that these conditions hold whenever $q_{k}(t)$ is replaced by a continuous function $Q_{k}(t)$ satisfying $\left|Q_{k}(t)\right|<q_{k}(t)+\left(\eta_{*}-\eta\right) / n T$, where $\eta_{*}=\eta_{0}$, $\eta_{1}, \eta_{2}$, respectively. The proof for the general case (4.1)-(4.3) is similar. 


\section{APPENDIX}

Let $y^{k}=\left(y_{1}^{k}, \ldots, y_{m(k)}^{k}\right)$ be a real Euclidean vector of dimension $m(k)$ and $y=\left(y^{1}, \ldots, y^{N}\right)=\left(y_{1}^{1}, \ldots, y_{m(N)}^{N}\right)$ the corresponding Euclidean vector of dimension $M=m(1)+\cdots+m(N)$. Consider a linear differential equation

$$
y^{\prime}=A(t) y,
$$

where $A(t)$ is an $M \times M$ matrix with real continuous entries for $0 \leqq t<T(\leqq \infty)$. Let $\kappa$ be fixed, $1 \leqq \kappa \leqq N$. Assume that there exists continuous functions $\psi_{1}(t) \geqq 0, \ldots$, $\psi_{n}(t) \geqq 0$ and $\alpha_{1}(t), \ldots, \alpha_{x}(t)$ and $\alpha^{x}(t), \ldots, \alpha^{N}(t)$ such that $(1)$ implies

$$
\begin{array}{ll}
y^{k} \cdot y^{k^{\prime}} \leqq \alpha_{k}(t)\left|y^{k}\right|^{2}+\psi_{k}(t)\left|y^{k}\right| \cdot|y| & \text { for } k=1, \ldots, \kappa, \\
y^{k} \cdot y^{k^{\prime}} \geqq \alpha^{k}(t)\left|y^{k}\right|^{2}-\psi_{k}(t)\left|y^{k}\right| \cdot|y| & \text { for } k=\kappa, \ldots, N .
\end{array}
$$

Assume that the integrals

(4) $\sigma_{k}(t)=\int_{0}^{t}\left[\psi_{k}(s)+\psi_{k}(s)\right]\left\{\exp \int_{s}^{t}\left[\alpha_{k}(\rho)-\alpha^{\kappa}(\rho)\right] d \rho\right\} d s$ for $k=1, \ldots, \kappa-1$,

$$
\sigma_{k}(t)=\int_{t}^{T}\left[\psi_{k}(s)+\psi_{k}(s)\right]\left\{\exp -\int_{t}^{s}\left(\alpha^{k}(\rho)-\alpha_{k}(\rho)\right] d \rho\right\} d s
$$

for $k=\kappa+1, \ldots, N$,

exist and are bounded for $0 \leqq t<T$. Suppose finally that there exist positive constants $\gamma, \eta$ with the properties that

$$
\begin{gathered}
\gamma \sigma_{k}(t) \leqq \eta \text { for } 0 \leqq t<T, k \neq \kappa, \\
{\left[1+(N-1) \eta^{2}\right]^{1 / 2} \max (1, \eta)<\gamma .}
\end{gathered}
$$

THEOREM (*). Under the conditions enumerated above, there exists an $m(1)+\cdots$ $+m(\kappa)$ parameter family of solutions of (1) such that, for $0 \leqq t<T$, we have $y^{\kappa}(t) \neq 0$ and

$$
\left|y^{k}(t)\right| \leqq \gamma \sigma_{k}(t)\left|y^{x}(t)\right| \text { for } k \neq \kappa .
$$

REMARK 1. The proof will show that one obtains a solution of (1) satisfying (8) for which one can assign an arbitrary partial set of initial conditions $y^{k}(0)$, $k=1, \ldots, \kappa$ such that $y^{\kappa}(0) \neq 0$ and $\left|y^{k}(0)\right| /\left|y^{x}(0)\right|$ is small for $k=1, \ldots, \kappa-1$.

REMARK 2. Theorem $\left({ }^{*}\right)$ has an analogue if the components of $y$ and the entries of $A(t)$ are complex-valued. It is only necessary to replace $y^{k} \cdot y^{k^{\prime}}$ by $\operatorname{Re} \bar{y}^{k} \cdot y^{k^{\prime}}$ in (2), (3).

REMARK 3. Note that there is no assumption about the signs of the functions $\alpha_{k}, \alpha^{k}, \alpha^{x}-\alpha_{k}, \alpha^{k}-\alpha_{k}$.

For particular cases of this theorem, see [2, pp. 284-290; especially, Lemmas 4.1, 4.2 and Exercise 4.3].

Proof. Theorem $\left({ }^{*}\right)$ will be deduced from a result of Ważewski [15]. We shall use the formulation and notation of [2, Theorem 3.1, p 282]. In order to be able to use 
this theorem, we shall suppose that $\psi_{k}(t)>0$ for $0<t<T$ and prove the existence of solutions satisfying $y^{x}(t) \neq 0$ and (8) for $t_{0} \leqq t<T$, where $t_{0}$ is an arbitrary point of $(0, T)$. The passage to the case $\psi(t) \geqq 0$ and $t_{0}=0$ will be clear.

Let $\Omega$ be the open $(t, y)$-set $\Omega=\{(t, y): 0<t<T, y \neq 0$ arbitrary $\}$. Define an open subset by

$$
\Omega^{0}=\left\{(t, y) \in \Omega: u_{j}(t, y)<0, v_{k}(t, y)<0\right\}
$$

for $1 \leqq k<\kappa<j \leqq N$, where

$$
\begin{gathered}
u_{b}(t, y)=\left|y^{b}\right|^{2}-\gamma^{2} \sigma_{b}^{2}(t)\left|y^{\kappa}\right|^{2} \text { for } b=\kappa+1, \ldots, N, \\
v_{a}(t, y)=\left|y^{a}\right|^{2}-\gamma^{2} \sigma_{a}^{2}(t)\left|y^{\kappa}\right|^{2} \text { for } a=1, \ldots, \kappa-1 .
\end{gathered}
$$

Correspondingly, define the subsets of $\Omega$

$$
\begin{aligned}
& U_{b}=\left\{u_{b}(t, y)=0, u_{j}(t, y) \leqq 0, v_{k}(t, y) \leqq 0 \text { for } 1 \leqq k<\kappa<j \leqq N\right\}, \\
& V_{a}=\left\{v_{a}(t, y)=0, u_{j}(t, y) \leqq 0, v_{k}(t, y) \leqq 0 \text { for } 1 \leqq k<\kappa<j \leqq N\right\},
\end{aligned}
$$

where $a=1, \ldots, \kappa-1$ and $b=\kappa+1, \ldots, N$.

If $f(t, y)$ is a function of class $C^{1}$, let $\dot{f}(t, y)$ be the trajectory derivative of $f$ relative to (1); i.e.,

Then we can verify

$$
\dot{f}(t, y)=\partial f \mid \partial t+\left(\operatorname{grad}_{y} f\right) \cdot A(t) y .
$$

$$
\begin{array}{ll}
\dot{v}_{a}(t, y)>0 & \text { for }(t, y) \in V_{a}, a=1, \ldots, \kappa-1, \\
\dot{u}_{b}(t, y)<0 & \text { for }(t, y) \in U_{b}, b=\kappa+1, \ldots, N .
\end{array}
$$

For example, in order to obtain (11), note that

$$
\dot{u}_{b}=2\left(y^{b} \cdot y^{b^{\prime}}-\gamma^{2} \sigma_{b}^{2} y^{\alpha} \cdot y^{\kappa^{\prime}}-\gamma^{2} \sigma_{b} \sigma_{b}^{\prime}\left|y^{\alpha}\right|^{2}\right) .
$$

Using (2) and (3), it is seen that

$$
\frac{1}{2} \dot{u}_{b} \geqq \alpha^{b}\left|y^{b}\right|^{2}-\psi_{b}(t)\left|y^{b}\right| \cdot|y|-\gamma^{2} \sigma_{b}^{2}\left(\alpha_{x}\left|y^{\alpha}\right|^{2}+\psi_{x}(t)\left|y^{x}\right| \cdot|y|\right)-\gamma^{2} \sigma_{b} \sigma_{b}^{\prime}\left|y^{x}\right|^{2} .
$$

For $(t, y) \in U_{b}$, we have $\left|y^{b}\right|=\gamma \sigma_{b}\left|y^{x}\right|$ and

$$
|y| \leqq\left|y^{x}\right|\left(1+\sum_{j \neq x} \gamma^{2} \sigma_{j}^{2}\right)^{1 / 2} \leqq c\left|y^{x}\right|,
$$

where, by (6), $c=\left[1+(N-1) \eta^{2}\right]^{1 / 2}>0$. Thus we have

$$
\frac{1}{2} \dot{u}_{b} \geqq \gamma^{2} \sigma_{b}\left|y^{x}\right|^{2}\left\{\left(\alpha^{b}-\alpha_{x}\right) \sigma_{b}-\sigma_{b}^{\prime}-(c / \gamma)\left[\psi_{b}+\psi_{x} \gamma \sigma_{b}\right]\right\} .
$$

Note that, by (5),

hence, for $(t, y) \in U_{b}$,

$$
\sigma_{b}^{\prime}=-\left(\psi_{b}+\psi_{x}\right)+\left(\alpha^{b}-\alpha_{k}\right) \sigma_{b}
$$

$$
\frac{1}{2} \dot{u}_{b} \geqq \gamma \sigma_{b}\left|y^{x}\right|^{2}\left\{(\gamma-c) \psi_{b}+\psi_{x}\left(\gamma-c \gamma \sigma_{b}\right)\right\} .
$$

Thus $\frac{1}{2} \dot{u}_{b}>0$ if $\gamma>c$ and $\gamma>c \gamma \sigma_{b}$, while $\gamma>c \gamma \sigma_{b}$ if $\gamma>c \eta$. (Note that $y^{x} \neq 0$, for 
otherwise $(t, y) \in U_{b} \subset \Omega$ implies that $y=0$, which cannot hold for $(t, y) \in \Omega$.) Similarly (10) is verified.

Thus, in the terminology of [2, pp. 281-282], $\Omega^{0}$ is a $(u, v)$-subset of $\Omega$ and, by a theorem of Ważewski [13] (cf. [2, Lemma 3.1, p. 281]), the set of egress points $\Omega_{e}^{0}$ of $\Omega^{0}$ consists only of strict egress points and

$$
\Omega_{e}^{0}=\bigcup_{b=x+1}^{N} U_{b}-\bigcup_{a=1}^{x-1} V_{a} .
$$

If $0<t_{0}<T$, let $y^{1}\left(t_{0}\right), \ldots, y^{x}\left(t_{0}\right)$ be arbitrary vectors satisfying $y^{x}\left(t_{0}\right) \neq 0,\left|y^{a}\left(t_{0}\right)\right|$ $<\gamma \sigma_{a}\left(t_{0}\right)\left|y^{x}\left(t_{0}\right)\right|$ for $a=1, \ldots, \kappa-1$. Let $S$ be the set of points $(t, y)=\left(t_{0}, y^{1}\left(t_{0}\right), \ldots\right.$, $\left.y^{x}\left(t_{0}\right), y^{x+1}, \ldots, y^{N}\right) \in \Omega$ satisfying $\left|y^{b}\right| \leqq \gamma \sigma_{b}\left(t_{0}\right)\left|y^{x}\left(t_{0}\right)\right|$ for $b=\kappa+1, \ldots, N$. Topologically, $S$ is a ball of dimension $m(\kappa+1)+\cdots+m(N)$ and $S \cap Q_{e}^{0}$ is its boundary. Thus $S \cap \Omega_{e}^{0}$ is not a retract of $S$. But $S \cap \Omega_{e}^{0}$ is a retract of $\Omega_{e}^{0}$. In fact, a retraction is given by the map $\pi: \Omega_{e}^{0} \rightarrow S \cap \Omega_{e}^{0}$ defined by $\pi(t, y)=\left(t_{0}, z\right)$, where $z^{a}=y^{a}\left(t_{0}\right)$ for $a=1, \ldots, \kappa$ and $z^{b}=y^{b} \sigma_{b}\left(t_{0}\right)\left|y^{\kappa}\left(t_{0}\right)\right| / \sigma_{a}(t)\left|y^{x}\right|$ for $b=\kappa+1, \ldots, N$. The map $\pi$ is continuous (since $y \neq 0$, hence $y^{x} \neq 0$, on $\Omega_{e}^{0}$ and $\left.\sigma_{b}(t)>0\right), \pi\left(\Omega_{e}^{0}\right) \subset S \cap \Omega_{e}^{0}$ and $\pi \mid S \cap \Omega_{e}^{0}$ is the identity.

By a theorem of Ważewski [13] (cf. [2, Theorem 3.1, p. 282]), it follows that there exists a point $\left(t_{0}, y\left(t_{0}\right)\right) \in S$ such that the solution of (1) determined by this initial condition is in $\Omega^{0}$ for $t_{0} \leqq t<T$, i.e., satisfies (8). This proves Theorem (*).

REMARK. It is clear from the proof of this theorem that the conclusion is valid if the functions (4)-(5) and conditions (6)-(7) are replaced by a set of functions $\sigma_{a}(t)$ and $\sigma_{b}(t), 1 \leqq a<\kappa<b \leqq N$, which are nonnegative, continuously differentiable for $0 \leqq t<T$, and satisfy the following system of differential inequalities

$$
\sigma_{b}^{\prime}=\left[\alpha^{b}(t)-\alpha_{x}(t)\right] \sigma_{b}-\chi_{b}(t), \quad \sigma_{a}^{\prime}=\left[\alpha_{a}(t)-\alpha^{\kappa}(t)\right] \sigma_{a}+\chi_{a}(t),
$$

where $\chi_{a}, \chi_{b}$ are continuous functions satisfying

$$
\gamma \chi_{k}>\left(1+\gamma^{2} \sum_{j \neq \kappa} \sigma_{j}^{2}\right)^{1 / 2}\left[\psi_{k}+\psi_{\kappa} \gamma \sigma_{k}\right], \quad k \neq \kappa .
$$

\section{REFERENCES}

1. P. Hartman, Unrestricted n-parameter families, Rend. Circ. Mat. Palermo (2) 7 (1958), 123-142.

2. - Ordinary differential equations, Wiley, New York, 1964.

3. P. Hartman and A. Wintner, $A$ criterion for the non-degeneracy of the wave equation, Amer. J. Math. 71 (1949), 206-213.

4. _- On an oscillation criterion of Liapounoff, Amer. J. Math. 73 (1951), 885-890.

5. —_, Asymptotic integrations of linear differential equations, Amer. J. Math. 77 (1955), 45-87.

6. D. B. Hinton, Disconjugate properties of a system of differential equations, J. Differential Equations 2 (1966), 420-437.

7. M. Hukuhara, On the zeros of solutions of linear ordinary differential equations, Sûgaku 15 (1963), 108-109 (Japanese). Cf. Math. Reviews 29 (1965), 709, \#3704, and reference there to M. Nagumo, T. Sato and M. Tumura. 
8. A. Lasota and Z. Opial, L'existence et l'unicité des solutions du problèmes d'interpolation pour l'équation différentielle ordinaire d'ordre n, Ann. Polon. Math. 15 (1964), 253-271.

9. A. Ju. Levin, On some boundary value problems, Naučn. Dokl. Vysš. Skoly Fiz.-Mat. Nauki 1958, No. 5, 34-37. (Russian)

10. - Some estimates of a differentiable function, Dokl. Akad. Nauk SSSR 138 (1961), 37-38 = Soviet Math. Dokl. 2 (1961), 523-524.

11. Z. Nehari, On an inequality of Lyapunov, pp. 256-261, Studies in Mathematical Analysis and Related Topics, Stanford Univ. Press, Stanford, Calif., 1962.

12. — Disconjugacy criteria for linear differential equations, Carnegie Inst. Tech. Rep., Pittsburgh, Pa., 1967.

13. Z. Opial, On a theorem of O. Aramă, J. Differential Equations 3 (1967), 88-91.

14. G. Pólya, On the mean value theorem corresponding to a given linear homogeneous differential equation, Trans. Amer. Math. Soc. 24 (1922), 312-324.

15. T. Ważewski, Sur un principe topologique de l'examen de l'allure asympotique des intégrales des équations différentielles ordinaires, Ann. Soc. Polon. Math. 20 (1947), 279-313.

16. A. Wintner, On the non-existence of conjugate points, Amer. J. Math. 73 (1951), 368-380.

THE JOHNS HOPKINS UNIVERSITY,

BALTIMORE, MARYLAND 